\title{
Germanica
}

contemporain : jeux intermédiaux, modes de transfert, adaptations

\section{„Die Bilder wollen automatisch anders geschnitten werden“}

Die Verfilmung von Daniel Kehlmanns Die Vermessung der Welt

"Die Bilder wollen automatisch anders geschnitten werden" - The film adaptation of Daniel Kehlmann's Die Vermessung der Welt

"Die Bilder wollen automatisch anders geschnitten werden" - L'adaptation cinématographique de Die Vermessung der Welt de Daniel Kehlmann

Jan Rhein

\section{(2) OpenEdition}

\section{Journals}

Édition électronique

URL : http://journals.openedition.org/germanica/2287

DOI : $10.4000 /$ germanica.2287

ISSN : 2107-0784

Éditeur

Université de Lille

Édition imprimée

Date de publication : 30 décembre 2013

Pagination : 181-195

ISBN : 9782913857322

ISSN : 0984-2632

Référence électronique

Jan Rhein, " „Die Bilder wollen automatisch anders geschnitten werden“ », Germanica [Online], 53 |

2013, Online erschienen am: 30 Dezember 2016, abgerufen am 06 Oktober 2020. URL : http:// journals.openedition.org/germanica/2287 ; DOI : https://doi.org/10.4000/germanica.2287 


\title{
„Die Bilder wollen automatisch anders geschnitten werden“" \\ Die Verfilmung von Daniel Kehlmanns Die Vermessung der Welt
}

\author{
Jan RHEIN \\ Université de Nantes
}

\begin{abstract}
Nicht immer vereinfacht es den künstlerischen Prozess, wenn Literaten an der Verfilmung ihrer Bücher mitarbeiten. Vladimir Nabokov, einer der für Daniel Kehlmann einflussreichsten Autoren und der "treueste Hausdämon"1 seines Werks, schrieb das Drehbuch zur Verfilmung des Romans Lolita selbst. Es war so umfangreich, dass ein mehr als siebenstündiger Film daraus hätte werden können, und wurde von Stanley Kubrick, der es komplett überarbeitete, als unverfilmbar bezeichnet ${ }^{2}$. Nabokov zeigte sich von der Verfilmung enttäuscht:

The modifications, the garbling of my best little finds, the omission of entire scenes, the addition of new ones, and all sorts of other changes may not have been sufficient to erase my name from the credit titles but they certainly made the picture as unfaithful to the original script as an American poet's translation from Rimbaud or Pasternak ${ }^{3}$.
\end{abstract}

1. - Markus Gasser, Das Königreich im Meer. Daniel Kehlmanns Geheimnis, Göttingen, Wallstein, 2010, S. 99.

2. - Vgl. Georg Seeßlen und Fernand Jung, Stanley Kubrick und seine Filme, Marburg, Schüren, 1999, S. 118-123. S. 13.

3. - Vladimir Nabokov, Lolita. A screenplay, New York/Toronto, Vintage, 1997,

GERMANICA, 2013, LIII, p. 181 à 195. 
Mit Nabokovs doppelbödigem Roman hat Die Vermessung der Welt $(1995)^{4}$ nicht die Skandalträchtigkeit gemein, wohl aber den Umstand, dass, wie Kehlmann selbst vermutet, hier wie dort „die Bestsellerliste [den Lesern] ein Buch aufgezwungen [habe], das ihren Wünschen möglicherweise gar nicht [entspreche]"5. Von den großen deutschsprachigen Prosaerfolgen der zwei letzten Jahrzehnte - sie handeln von einem von guten Düften besessenen Frauenmörder (Patrick Süskinds Das Parfüm), der Liebesgeschichte zwischen ehemaliger KZ-Aufseherin und jungem Schöngeist (Bernhard Schlinks Der Vorleser) und dem Intimleben einer Jugendlichen (Charlotte Roches Feuchtgebiete) - ist die im Konjunktiv-Stil verfasste Geschichte zweier Wissenschaftler im ausgehenden 18. Jahrhundert sicherlich der ungewöhnlichste. Für Kehlmann überdeckt der Erfolg seines Romans, dass dieser ,in seiner Erzählweise ein Experiment war. [Er] geht gegen genau das, was man sonst unter einem historischen Roman versteht, er ist viel knapper, ironischer, in gewisser Weise wegwerfender"6.

Das Werk, zugleich literarisches Experiment und Weltbesteller, mag in seinem Ansatz an andere postmoderne historische Erfolgsromane wie Umberto Ecos Il nome della rosa oder Das Parfum, beide mit großem Erfolg verfilmt, denken lassen. Anders als diese beiden Beispiele verzichtet Die Vermessung der Welt jedoch auf eine stringente Handlung und äußere Konflikte. Regisseur Detlev Buck beschreibt es so: „Es gibt keinen Antagonisten, keinen Showdown, nicht den Wendepunkt, das ganze dramaturgische Geschirr gibt es nicht.“7

Die Verfilmung des Buchs - einerseits aufwändig aufgrund seiner exotischen Handlungsorte und seiner historischen Dimension, andererseits dramaturgisch anspruchsvoll - kam erst im Jahr 2012 in die deutschen $\mathrm{Kinos}^{8}$, nachdem der Roman bereits als Vorlage für ein von Alexander Schuhmacher inszeniertes Hörspiel und eine Theaterinszenierung von Dirk Engler am Staatstheater Braunschweig gedient hatte. Der Film wurde als deutsch-österreichische Koproduktion finanziert. Die Humboldt-Szenen wurden größtenteils in Ecuador

4. - Daniel Kehlmann, Die Vermessung der Welt. Roman, Reinbek bei Hamburg, Rowohlt, 2005. (Im Text zitiert mit der Abkürzung VM und Seitenangabe)

5. - Ders., ,Die Katastrophe des Glücks. Dankesrede zur Verleihung des WELTLiteraturpreises“, in: ders., Lob. Über Literatur, Reinbek bei Hamburg, Rowohlt, 2011, S. 169-178, hier S. 174.

6. - Willi Winkler, ,Ein Fenster in die Vergangenheit. Daniel Kehlmann und Detlev Buck im Gespräch“, in: Wenka v. Mikulicz u. Michael Töteberg (Hrsg.) Die Vermessung der Welt. Das Buch zum Film, Reinbek bei Hamburg, Rowohlt, 2012, S. 145-157, hier S. 153.

7. - Ebd., S. 154.

8. - Die Vermessung der Welt, Regie: Detlev Buck, Deutschland/Österreich, Boje Buck Produktion/Lotus Film, 2012. (Zitiert im Text mit der Abkürzung V und Zeitangabe) 
gedreht, die Gauß-Szenen in Görlitz. Mit Detlev Buck hatte sich ein Regisseur des Stoffs angenommen, dessen frühere Filme wie Karniggels oder Wir können auch anders für trockenen, eigenbrötlerischen Humor stehen, der vor allem ein norddeutsches oder großstädtisches Programmkinopublikum erreichte. Seit einigen Jahren kann er als einer der erfolgreichsten deutschen Regisseure gelten, der sich inzwischen immer mehr dem Hollywood-Kino annähert und sich etwa in der Travestie-Komödie Rubbeldiekatz klassischer Screwball-Elemente bedient.

Trotz der Bekanntheit der literarischen Vorlage und der bekannten Namen Bucks und Kehlmanns war der Film weder ein Kritiker- noch ein Publikumserfolg. Im Jahr 2012 lag er mit knapp 600.000 Zuschauern in Deutschland zwar weit vor etwa der Verfilmung von Marlen Haushofers Die Wand, blieb jedoch beispielsweise hinter der Adaptation von Wladimir Kaminers Russendisko zurück. Der erfolgreichste deutsche Film des Jahres, Türkisch für Anfänger, erreichte im selben Jahr mehr als zwei Millionen Zuschauer, die französische Komödie Intouchables gar weit über sieben Millionen. Von der Kritik wurde der Film fast durchgängig schlecht aufgenommen. „Schöne Vögel, lange Formeln. Bucks Film ist eine Bilderflut, aber kein Kino“, schrieb Die Zeit, „,Buck [huldige] einem erzählerischen Obskurantismus“, bemängelte die taz, im Standard hieß es, der Film gehe ,mit stilistischem Sperrfeuer auf die Vorlage los und [treffe] selten eine richtige Tonlage“"9.

Über die Gründe für den Erfolg des Romans, dessen gebundene Ausgabe sich im Besitz von etwa 1,1 Prozent aller Deutschen ,,inklusive Säuglinge[n] und Sieche[n]" 10 befinden soll, ist viel spekuliert worden ${ }^{11}$. Ebenso viele Mutmaßungen ließen sich über den Misserfolg des Films anstellen. Eine Erklärung für die Eigenheiten des Films findet sich jedoch eher, wenn man diese als bewusst gewählte, narrative Strategien betrachtet und eine Analyse des Films ,entlang des Romans" und im Kontext des Werks Kehlmanns unternimmt. Dessen enge Beteiligung an der Umsetzung lässt sich als „Autorisierung“ der Verfilmung verstehen: Anders als Kubrick und Nabokov treten Buck und er als Ko-Drehbuchautoren auf. Weiterhin hat Kehlmann sich in einer

9. - Eine Sammlung von Filmrezensionen findet sich auf http://www.film-zeit. de/Film/22629/DIE-VERMESSUNG-DER-WELT/Kritik/, die Eintrittszahlen sind der Seite http://www.insidekino.com/DJahr/IM12.htm entnommen.

10. - Marius Meller, „Die Krawatte im Geiste“, in: Merkur, 2007, 61, S. 248-252, hier S. 250.

11. - Vgl. stellvertretend Heinz-Peter Preußler, „Zur Typologie der Zivilisationskritik. Was aus Daniel Kehlmanns Roman Die Vermessung der Welt einen Bestseller werden ließ“", in: Fabrizio Cambi (Hrsg.), Gedächtnis und Identität. Die deutsche Literatur nach der Vereinigung, Würzburg, Königshausen \& Neumann, 2008, S. 111-124. 
Nebenrolle in den Film hineingeschrieben. Er spielt einen Staatssekretär des Königs, im Drehbuch als „Der unheimliche Mann“ benannt ${ }^{12}$, und spricht den Off-Erzähler im Film. Gemeinsam mit Buck beteiligte er sich an einem langen Gespräch im „Buch zum Film“ und sprach einen Audiokommentar zur DVD des Films ein. Diese enge Beteiligung des Autors am Entstehungsprozess und auch der Vermarktung des Films erlaubt es, ästhetische Entscheidungen nicht nur auf ihre dramaturgische Funktion hin zu betrachten, sondern sie auch als intentionale „Erzählentscheidungen“ Kehlmanns zu beleuchten.

\section{Vereinfachung und Verkomplizierung}

Bereits an der ersten Passage des Romans lassen sich grundsätzliche Unterschiede zwischen Buch und Film festmachen:

Im September 1828 verließ der größte Mathematiker des Landes zum erstenmal seit Jahren seine Heimatstadt, um am Deutschen Naturforscherkongreß in Berlin teilzunehmen. Selbstverständlich wollte er nicht dorthin. Monatelang hatte er sich geweigert, aber Alexander von Humboldt war hartnäckig geblieben, bis er in einem schwachen Moment und in der Hoffnung, der Tag käme nie, zugesagt hatte.

Nun also versteckte sich Professor Gauß im Bett. Als Minna ihn aufforderte aufzustehen, die Kutsche warte und der Weg sei weit, klammerte er sich ans Kissen und versuchte seine Frau zum Verschwinden zu bringen, indem er die Augen schloß. (VM, S. 7)

Der erste Unterschied zur Verfilmung betrifft den Erzählgestus: Die Gattung des historischen Romans klingt an und wird wieder zertrümmert, der hohe Ton einer Intellektuellenbiographie kontrastiert spätestens im zweiten Absatz mit der dargestellten Sonderbarkeit des Wissenschaftlers und benennt etwas, das ein Biograph nicht wissen kann; für diese „Ironie der Haltung“, wie Kehlmann das Verfahren in seiner Poetikvorlesung ${ }^{13}$ nennt, findet sich keine direkte Übertragung in die Filmsprache. Man könnte hingegen von einer einem „Historienfilm“ gegenlaufenden Ironie des Bildes sprechen, etwa, wenn der distinguierte Humboldt in Bucks Film in seiner preußischen Uniform durch den Urwald stapft, oder Gauß seiner zukünftigen Frau Johanna seine Liebe erklärt, während im Hintergrund jemand gerupfte Gänse vorbeiträgt.

Der zweite Unterschied, der anklingt, betrifft das Weltbild. Der Roman führt die Namen seiner Protagonisten schon in den ersten

12. - Daniel Kehlmann u.a., ,Die Vermessung der Welt. Der Film“, in: Wenka v. Mikulicz u. Michael Töteberg (Hrsg.), Die Vermessung der Welt. Das Buch zum Film, a.a.O., S. 16-130, hier S.116.

13. - Daniel Kehlmann, „Diese sehr ernsten Scherze. Zwei Poetikvorlesungen“, in: ders., Lob. Über Literatur, a.a.O., S. 125-168, hier S. 147. 
Sätzen zusammen, stellt aber zugleich eine Distanz zwischen ihnen her. Humboldt und Gauß, deren Schicksale fragmentarisch geschildert werden, nähern sich im Lauf des Romans nur gelegentlich an, insbesondere über ihre Korrespondenzen, in welchen sie eine Art „Ferndialog“14 entspinnen: Gauß sendet Humboldt ,gute Wünsche sowie einige Formeln für die magnetischen Messungen, von denen Humboldt keine Zeile verstand“ (VM, S. 266). Humboldt antwortet, ,,[m]an müsse die Pläne ändern, [...] er könne nicht, wie er wolle. [...] Gauß legte traurig lächelnd den Brief weg. Zum erstenmal tat Humboldt ihm leid." (VM, S. 273-4). Die Annäherung zwischen beiden fällt bis zum Ende des Romans subtil aus, als Humboldt von seiner Russlandreise heimkehrt:

[W]ährend die ersten Vororte Berlins vorbeiflogen und Humboldt sich vorstellte, wie Gauß eben jetzt durch sein Teleskop auf Himmelskörper sah, deren Bahnen er in einfache Formeln fassen konnte, hätte er auf einmal nicht mehr sagen können, wer von ihnen weiter herumgekommen war und wer immer zu Hause geblieben. (VM, S. 293)

Entsprechend der Erkenntnis Gauß', dass ,[d]er Satz, daß zwei gegebene Parallelen einander niemals berührten, [..] nie beweisbar gewesen" (VM, S. 93) sei ${ }^{15}$, zeichnet der Roman die beiden Figuren also als Einzelgänger im luftleeren Raum, deren Schicksale unabhängig voneinander verlaufen, nicht ohne gewisse Annäherungspunkte zwischen beiden zuzulassen.

Der Film hingegen beginnt nach einem Prolog mit einer chronologischen Erzählung, weshalb die zitierte Eingangsszene erst im letzten Drittel des Films zu sehen ist. Dieser verschränkt die Schicksale der beiden Forscher immer wieder: Hier der junge Gauß in der Volksschule, dort die Humboldt-Brüder Latein lernend bei Hof. Einerseits werden so die Lebenswelten der beiden kontrastiert, andererseits aber auch eine Geschlossenheit der Handlung hergestellt. Nicht nur zeigt der Film die beiden Protagonisten parallel in ihrer Entwicklung, auch zahlreiche Bildmotive, wie eine erst durchs Zimmer des einen, dann des anderen fliegende Feder, verbinden die Welten beider Wissenschaftler. Auch die Montage suggeriert eine Geschlossenheit und Sinnhaftigkeit der erzählten Welt. So sind etwa mehrfach Großaufnahmen von schlüpfenden Schmetterlingen oder anderen Insekten als „Konnektoren“ zwischen einer Humboldt- und einer Gauß-Szene einmontiert, die keiner eindeutig zugeordnet werden können. Bei einer ersten Begegnung

14. - Barbara Sandig, „Spannende Dialoge im Konjunktiv: Kehlmanns ,Die Vermessung der Welt““, in: Literaturstil - sprachwissenschaftlich, Heidelberg, Winter, 2008, S. 275-293, hier S. 288.

15. - Vgl. Alexander Honold, „Ankunft in der Weltliteratur. Abenteuerliche Geschichtsreisen mit Ilija Trojanow und Daniel Kehlmann“, in: Neue Rundschau 2007, 118, 1, S. 82-104, hier S. 93-94 
beim Herzog von Braunschweig, welche im Buch nicht vorkommt, werfen sich die Knaben Gauß und Humboldt lange Blicke zu - es wird der Eindruck zweier Genies vermittelt, die sich erkennen (V 14:40). Diese bedeutungsvollen Blickwechsel stellen ein weiteres Leitmotiv dar, welches beispielsweise auch der ersten Begegnung von Gauß und seiner späteren Frau Johanna (V 34:40) eine Sinnhaftigkeit und Determiniertheit zuweist und der Humboldtschen Aussage entspricht, „die Welt [sei] ein Ganzes [...], alle Systeme [seien] ein System“ (V 01:05:17). Diese kontrastiert mit der Grundaussage des Romans, in dem es bezeichnenderweise nur heißt, die „Natur sei ein Ganzes“( $V M$, S. 117). Buck erklärt diesen Unterschied dramaturgisch:

Im Drehbuch hatten wir sowohl für Humboldt als auch für Gauß längere Passagen, wo man der jeweiligen Geschichte folgte, dann aber gemerkt, das geht nicht, weil man dann den anderen aus dem Blickfeld verliert. Wenn man Figuren zu lange weglässt, dann interessieren sie einen emotional nicht mehr so. Beim Roman ist das anders. Beim Lesen dauert es zwar länger, aber der Kopf schiebt es dichter zusammen. Die Bilder wollen automatisch anders geschnitten werden ${ }^{16}$.

Auch formal steht der Film im Gegensatz zur verknappten Konstruktion des Romans: Er ist, wie es in einer Spiegel-Rezension heißt, ,die vergnügungssüchtigste Filmversion, die einer deutschen Schullektüre je widerfahren ist"17 und schöpft die ganze Bandbreite filmischen Erzählens aus: Sie erstreckt sich von Halleffekten und einer kommentierenden Off-Stimme über Computeranimationen, extreme Nahaufnahmen, Ober- und Untersichten, stark nachkolorierte Bilder bis schließlich zum Einsatz von digitaler 3D-Technik.

Trifft also auf der Erzählebene die verbreitete Ansicht zu, die filmische Adaptation literarischer Werke bedeute meist auch Simplifizierung ${ }^{18}$, so lässt sich auf formaler Ebene eher das Gegenteil behaupten. Das Eine hat Auswirkungen auf das Andere: Der Film versucht, durch Stringenz in der Handlung einen Spannungsbogen zu erzeugen, die dominanten Bilder und Effekte hingegen verhindern eine stärkere Fokussierung auf die Geschichte und die Protagonisten und kreieren stattdessen ein kohärentes filmisches „Universum“.

16. - Willi Winkler, „Ein Fenster in die Vergangenheit. Daniel Kehlmann und Detlev Buck im Gespräch“, a.a.O., S. 152.

17. - Wolfgang Höbel, ,Zwei glorreiche Geisteshalunken“, in: Der Spiegel, 2012, 43, S. 132-133, hier S. 133.

18. - Vgl. Wolfgang Budecke u. Jörg Hienger, „Verfilmte Literatur. Probleme der Transformation und der Popularisierung“, in: LiLi, 1979, 9, S. 12-29, hier S. 12-13. 


\section{Vermessung und Vermessenheit}

Diese „Bilderflut“ des Films rückt einen Aspekt in den Vordergrund, welcher den gesamten Roman durchzieht und zu einem großen Teil für die von Kehlmann beschriebene „Ironie der Haltung“ verantwortlich ist: die Diskrepanz zwischen Vermessung und Vermessenheit, zwischen Pragmatismus und Verwirrung. Beide Pole fasst Humboldt am Ende des Romans zusammen, wenn er davon spricht, ,ein ungeheures Werk voller Tatsachen, jede Tatsache der Welt“, den ,ganze[n] Kosmos noch einmal“ aufzuschreiben, ,allerdings entkleidet von Irrtum, Phantasie, Traum und Nebel.“ (VM, S. 293)

Beide Figuren können im Roman nicht immer ihren Sinnen trauen, Humboldt, wenn er „Spiegelungen von Vögeln übers Wasser“ streichen sieht, ,selbst wenn der Himmel leer war“ (VM, S. 110), Gauß, wenn er sich an den Tod seiner Frau in vier verschiedenen Versionen erinnert: „Es kam ihm vor, als wäre die Zeit vor- und zurückgeschnellt, als hätten sich mehrere Möglichkeiten eröffnet und gegenseitig wieder ausgelöscht" (VM, S. 161). Gauß' Wahrnehmung kollidiert mit der Wirklichkeit, doch auch an der Mathematik zweifelt er noch. Die Erkenntnis, ,der Raum sei faltig, gekrümmt und sehr seltsam“ (VM, S. 96), ist noch eine Ahnung, die er mit niemandem teilen kann, und steht nicht für ,reine Vernunft“, sondern für Isolation und Weltzweifel ${ }^{19}$.

Das doppeldeutige Wort der „Vermessung“ 20 wird im Vorspann des Films durch einen Halleffekt besonders unterstrichen und betont das von Gauß empfundene ,verrückte“, „krumme“ Land als eine Art Grundkonstante des Films. Es schlägt sich in verschobenen Perspektiven und Ober- oder Untersichten nieder, aber auch in einer Kulisse, in der der Zuschauer gelegentlich die räumliche Orientierung verliert. Eine Szene mit Gauß in einem Bordell ist in Aufsicht gefilmt. In der folgenden Szene wird Gauß vom in Untersicht gefilmten Militaristen herbeizitiert und kommt - entgegen der Erwartung des Zuschauers - eine Treppe herunter. Mit derartigen Effekten wird eine Welt suggeriert, in der die Grenzen zwischen verschiedenen Lebensbereichen verschwimmen und es keine verlässlichen Konstanten gibt.

Der von Humboldt bereiste lateinamerikanische Urwald ist als Ort des europäischen Wahnsinns angelegt. Kulturelle Fremdheit und misslungene Kommunikation werden dabei mit kolonialer Vermessenheit zusammengeführt. Das bereits im Roman präsente Bild von „Aguirre de[m] Wahnsinnigen, der seinem König abgeschworen und sich selbst

19. - Mark M. Anderson, „,Der vermessene Erzähler. Mathematische Geheimnisse bei Daniel Kehlmann“, in: Text+Kritik, 2008, 177, S. 58-67, hier S. 63.

20. - Vgl.zu „Vermessung“ und „Vermessenheit“ Sean Moore Ireton, „Lines and crimes of demarcation. Mathematizing nature in Heidegger, Pynchon, and Kehlmann", in: Comparative literature, 2011, 63, 2, S. 142-160, hier S. 142. 
zum Kaiser ernannt hatte" (VM, S. 22) wird weiterentwickelt. Eine bezeichnende Szene lässt an Joseph Conrads Heart of Darkness bzw. dessen Verfilmung Apocalypse Now denken: Ein Pater herrscht brutal in einer Enklave am Orinoko über die Einheimischen, wo Humboldt den folgenden Dialog mit ihm führt: ,, Sind Sie Jesuit?‘ - , Sie wissen doch, dass die Jesuiten aus diesem Land vertrieben worden sind. Es gibt sie hier nicht mehr.“ - ,So dachte ich. Also sind Sie keiner?` - ,Das habe ich nicht gesagt"“ (V 39:40).

Gelegentlich setzt der Film für die im Roman sprachlich umgesetzte Verwirrung andere Erzählstrategien ein. Als Humboldt und Bonpland im Buch während der Besteigung des Chimborazo unter Sauerstoffmangel leiden, führen sie das folgende Gespräch: „Man könnte, sagte Bonpland, auch einfach behaupten, man wäre oben gewesen. Humboldt sagte, er wolle das nicht gehört haben. Er habe das auch nicht gesagt. Das sei der andere gewesen! Überprüfen könne es ja keiner, sagte Humboldt nachdenklich. Eben, sagte Bonpland. Er habe das nicht gesagt, rief Humboldt. Was gesagt, fragte Bonpland. Sie sahen einander ratlos an“ (VM, S. 177-178).

In dieser oft zitierten Szene geht es nicht - wie von manchen Kritikern herausgelesen ${ }^{21}$ - nur um den Geltungsdrang des Gipfelstürmers, sondern um eine Depersonalisierung, bei der sich beide Persönlichkeiten ,jeweils in drei spalten und in drei Stimmen“22 sprechen. Für Kehlmann selbst ist sie eine Darstellung des Dilemmas der Weimarer Klassik: „Weimars Gesandter in Macondo durchläuft [...] auf dem Rücken des Vulkans Wahnsinn, Übelkeit, Schwindel, Angst und Verwirrung - all das also, dessen Leugnung den Klassiker überhaupt erst definiert.“23

Der Film, der diese Szene dialogisch etwas verkürzt ebenfalls enthält, setzt die im Roman angedeutete Depersonalisierung bildlich um: Bonpland erschlägt Humboldt im Wahnsinn mit einem Stein, worauf dieser ihm auf die Schulter tippt und wieder lebendig hinter ihm steht: „,Cher ami. Aimé. Kommen Sie, wir müssen unten sein, bevor es dunkel wird. - ,Ich habe gedacht, ich hätte Sie getötet' - ,Cher ami. Man versteht so schlecht"“ (V 01:27:25). Die Verwirrung wird durch ein Schneegestöber, welches in Teilen das Geschehen verdeckt,

21. - Vgl. Klaus Zeyringer, ,Vermessen. Zur deutschsprachigen Rezeption der Vermessung der Welt“, in: Gunther Nickel (Hrsg.), Daniel Kehlmanns „Die Vermessung der Welt “. Materialien, Dokumente, Interpretationen, Reinbek bei Hamburg, Rowohlt, 2008, S. 78-94, hier S. 83.

22. - Ebd.

23. - Daniel Kehlmann, „Wo ist Carlos Montúfar?“, in: Gunther Nickel (Hrsg.), Daniel Kehlmanns „Die Vermessung der Welt“. Materialien, Dokumente, Interpretationen, a.a.O., S. 11-25, hier S. 20. (Zuerst in: Daniel Kehlmann, Wo ist Carlos Montúfar? Über Bücher, Reinbek bei Hamburg, Rowohlt, 2005, S. 9-27.) 
sowie eine Montage, die die Orientierung des Zuschauers im Bildraum erschwert, noch verstärkt.

Diese im Film prominenter in den Vordergrund gestellten Szenen des Wahnsinns und der Vermessenheit verlagern die Handlung hin zu vordergründigen Konflikten, die Hilflosigkeit der beiden Protagonisten basiert weniger stark als im Roman auf ihrer intellektuellen Ausgeschlossenheit oder kognitivem Versagen, sondern auch auf einer situativ umgesetzten und konkreten Hilflosigkeit - sei sie kulturell oder räumlich bedingt.

Der Film geht hier also über das Buch hinaus: Betrifft die „Vermessenheit" im Roman die beiden Protagonisten und das Verhältnis $\mathrm{zu}$ ihrer Umwelt, so illustriert der Film eine insgesamt verschobene Welt. Aus dem Roman kann man eine „Zivilisationskritik“ in dem Sinn herauslesen, als er von der „Konditionierung von Subjekten [handelt], die sich selbst, der Natur und den Anderen herum Gewalt antun, um sich selbst zu erhalten" ${ }^{24}$, der Film stellt die Zivilisation insgesamt grotesk dar.

\section{Mittelbarkeit}

Die im oben zitierten Auszug eingesetzte indirekte Rede, eines der markantesten Merkmale des Romans, verhindert eine Identifikation des Rezipienten mit den Protagonisten und stellt, hier wie in zahlreichen anderen Beispielen, eine „Scheindistanz“ 25 her: „Nicht gut sei das, sagte Humboldt. Sie seien von Wasser umgeben und säßen am höchsten Punkt" (VM, S. 141). Die in der Szene offensichtliche Gefahr erzeugt keine Spannung, sondern Komik, da sie nicht unmittelbar, sondern, wie durch einen Schleier, mittelbar dargestellt wird ${ }^{26}$.

Wie sich literarische Mittelbarkeit in andere Medien transformieren lässt, ist eine in den letzten Jahren verstärkt diskutierte Frage der Intermedialitätsforschung 27 , auf die der Film verschiedene Antworten gibt.

Insbesondere kommt hierbei dem Einsatz von digitaler 3D-Technik eine entscheidende Rolle zu. Nach Tim Burtons Alice in Wonderland ist Die Vermessung der Welt wohl die erste 3D-Literaturverfilmung, nach

24. - Vgl. Heinz-Peter Preußler, ,Zur Typologie der Zivilisationskritik. Was aus Daniel Kehlmanns Roman Die Vermessung der Welt einen Bestseller werden ließ“, a.a.O., S. 117.

25. - Daniel Kehlmann, „Wo ist Carlos Montúfar?“, a.a.O., S. 20.

26. - Vgl. Gerhard Kaiser, ,Erzählen im Zeitalter der Naturwissenschaft. Zu Daniel Kehlmanns Roman Die Vermessung der Welt“", in: Sinn und Form, 2010, 62, 1, S. 122-134, hier S. 122-123.

27. - Vgl. zur Übersicht Matthias Brütsch, „Ist Erzählen graduierbar? Zur Problematik transmedialer Narrativitätsvergleiche“, in: DIEGESIS, 2013, 2.1, S. 54-74. 
Wim Wenders' Pina und dem Kinderfilm Wickie und die starken Männer der dritte deutsche Film in 3D. Für Kehlmann stellt der Einsatz der Technik ,in gewisser Weise das Äquivalent für die indirekte Rede“28 dar.

In Die Vermessung der Welt wird der Effekt häufig zu dekorativen Zwecken eingesetzt, etwa zur Bebilderung prachtvoller Urwaldlandschaften. Der stereoskopische Filmblick übernimmt aber auch eine bedeutungskonstituierende Funktion, so etwa, wenn ein preuBischer Militarist Gauß mit dem Zeigefinger droht, er solle bald gute Ergebnisse liefern: „Du weißt, was eine Zahl ist, Junge. Eine Zahl ist kein Spielzeug. Eine Zahl ist der Durchmesser des schwarzen Loches zwischen den Augen eines sterbenden Soldaten.“ Die Szene hebt die Macht des Militärs hervor und verdeutlicht das tatsächliche Interesse, welches der Herzog von Braunschweig mit einem Stipendium an den jungen Gauß verfolgt, dessen Forschungsergebnisse militärischen Zwecken dienen sollen. Der Zeigefinger des Soldaten ragt als „before the screen"-Aufnahme aus der Leinwand (V 24:20) und bedroht auch den Zuschauer.

Umgekehrt wird 3D häufig eingesetzt, um eine Distanz zwischen dem Geschehen und dem Zuschauer herzustellen, etwa, wenn im Klassenzimmer des jungen Gauß der Staub in der Luft vor dem Geschehen liegt. In diesem Fall erzeugt der Effekt keine affektive Zuschauerreaktion, sondern stört eher die filmische Illusion. Der Wahrnehmungspsychologe Hinderk M. Emrich merkt an, „daß der Raum durch die 3D-Stereoprojektion formal überbetont und geradezu , aufgerissen“" 29 werden könne. Die räumliche „Überdeterminierung“ unterdrücke die kreative Leistung des Zuschauers, und aus „Genauigkeit allein [entstehe] nichts Wirkliches“30. Was Emrich negativ formuliert, kann im Film als bewusst eingesetztes Stilmittel verstanden werden, dem mittelbaren Erzählen gerecht zu werden.

Gleiches gilt wohl auch für einen weiteren Aspekt: Ist das Buch bereits reich an intertextuellen und intermedialen Anspielungen, in seinen Referenzen auf lateinamerikanische Autoren und Geistesgrößen der Weimarer Klassik sowie durch einen „Gastauftritt“ Louis Daguerres ( $V M$, S. 15), dessen Fotografieverfahren Gauß ungnädig gegenübersteht, so lässt sich der Film erst recht als intermediales Werk par excellence bezeichnen, welches neben zahlreichen Bezügen zu anderen Filmen ${ }^{31}$

28. - Willi Winkler, „Ein Fenster in die Vergangenheit. Daniel Kehlmann und Detlev Buck im Gespräch“, a.a.O., S. 154.

29. - Hinderk M. Emrich, „Tiefgreifende Veränderungen. Über reale Virtualität und die Eroberung des Raumes im Film“, in: Schnitt, 2010, 59, S. 16-19, hier S. 17.

30. - Ebd, S. 18.

31. - Im DVD-Kommentar und in dem Buch zum Film nennen Buck und Kehlmann ebenso Anspielungen auf Hollywoodklassiker wie Pulp Fiction, Apocalypse Now und Citizen Kane, wie auf Fellini- und Werner-Herzog-Filme oder Le fabuleux 
auch Medienkombinationen ${ }^{32}$ einsetzt: Die Filmbilder werden mit eingeblendeten Fotografien, Illustrationen, Formeln, Schriftzeichen angereichert, so etwa im Fall der Darstellung der Gaußschen Summenformel, und erzeugen einen Illusionsbruch.

Eine dritte Form von Mittelbarkeit ergibt sich aus der Präsenz des Autors als Erzähler im Film. Die Off-Stimme Kehlmannns, welcher kein ausgebildeter Sprecher ist, stellt einen ästhetischen Bruch innerhalb eines ansonsten technisch perfekten Filmuniversums dar; die Wiener Sprachmelodie und die weniger geschulte Artikulation verleihen dem Film eine gegenwärtige, fast dokumentarische Ebene - gelegentlich hat man den Eindruck, einen DVD-Audio-Kommentar zu hören. Kehlmann verkörpert hier den Erzähler, zugleich aber auch sich selbst.

\section{Narrative Vermessung}

Diese Präsenz des Autors erscheint bemerkenswert - zumal wenn man dem von Kehlmann geäußerten Bekenntnis zur ,Idee der klassischen Moderne“ glauben will, ,dass der Autor völlig hinter das Werk zurücktritt“33. Seine intellektuelle und öffentliche Beteiligung an dem Film rechtfertigt es, diesen als Bestandteil eines Werks zu betrachten, welches sich mit der Frage nach den Möglichkeiten des Erzählens beschäftigt, ist doch eine der versteckten Leitfragen des Romans nicht nur jene nach der mathematischen oder geographischen Vermessung der Welt, sondern auch nach der narrativen ${ }^{34}$. Beide Forscher könnten auch als Autoren verstanden werden, die ihre Welt neuschreiben: „Manchmal war ihm, als hätte er den Landstrich nicht bloß vermessen, sondern erfunden, als wäre er erst durch ihn Wirklichkeit geworden." (VM, S. 268) In diesem Sinn verkörpern Gauß und Humboldt nicht nur die wissenschaftlichen Gegensätze von Induktion und Deduktion, sondern auch zwei entgegengesetzte Schaffenskonzepte: „Ein Mann allein am Schreibtisch. Ein Blatt Papier vor sich, allenfalls noch ein Fernrohr, vor dem Fenster der klare Himmel. Wenn dieser Mann nicht aufgebe, bevor er verstehe. Das sei vielleicht Wissenschaft" (VM, S. 247), sagt Gauß. Dem gegenüber steht Humboldt, der die Welt erlebend ordnet:

„Er war in Neuspanien, Neugranada, Neubarcelona, Neuandalusien und den Vereinigten Staaten gewesen, hatte den natürlichen Kanal

destin d'Amélie Poulain.

32. - Vgl. Irina Rajewsky, Intermedialität, Tübingen/Basel, Francke, 2002, S. 19.

33. - Klaus Zeyringer und Stefan Gmünder, „Klassiker und Drecksäue. Daniel Kehlmann und Helmut Krausser im Gespräch“, in: Volltext, 2006, 1, S. 3, zitiert nach: Ottmar Ette, ,Alexander von Humboldt in Daniel Kehlmanns Welt“, in: HiN. Internationale Zeitschrift für Humboldt-Studien, 2012, XIII, 25, S. 40.

34. - Vgl. Gerhard Kaiser, ,Erzählen im Zeitalter der Naturwissenschaft. Zu Daniel Kehlmanns Roman Die Vermessung der Welt“, a.a.O., S. 123. 
zwischen Orinoko und Amazonas entdeckt, den höchsten Berg der bekannten Welt bestiegen, Tausende Pflanzen und Hunderte Tiere, manche lebend, die meisten tot, gesammelt, hatte mit Papageien gesprochen, Leichen ausgegraben, jeden Fluß, Berg und See auf seinem Weg vermessen, war in jedes Erdloch gekrochen und hatte mehr Beeren gekostet und Bäume erklettert, als sich irgendjemand vorstellen mochte." (VM, S. 19)

Es ist der Unterschied zwischen zwei Methoden derWelterschließung. Der ganze Roman steht somit einerseits im Zeichen der Diskussion, wie etwas beschrieben werden könne. Er ist andererseits durchzogen von einer Erzählkritik. Humboldt kritisiert insbesondere den historischen Roman: Es sei „ein albernes Unterfangen, wenn ein Autor, [...] eine schon entrückte Vergangenheit zum Schauplatz wähle." (VM, S. 27), Gauß hingegen bemängelt,

„Künstler vergäßen zu leicht ihre Aufgabe: das Vorzeigen dessen, was sei. Künstler hielten Abweichungen für eine Stärke, aber Erfundenes verwirre die Menschen, Stilisierung verfälsche die Welt. Bühnenbilder etwa, die nicht verbergen wollten, daß sie aus Pappe seien, englische Gemälde, deren Hintergrund in Ölsauce verschwimme, Romane, die sich in Lügenmärchen verlören, weil der Verfasser seine Flausen an die Namen geschichtlicher Personen binde.“ (VM, S. 221)

Diese Reflexion von Autorschafts- und Darstellungsformen, die Frage nach Wahrhaftigkeit von Literatur ist eine Grundkonstante des Gesamtwerks Kehlmanns, in welchem Die Vermessung der Welt ein Vor- und ein Nachleben besitzt. Der Roman fügt sich in einen erzählerischen Kosmos ein - er stellt die Frage nach Genie und Wahnsinn wie Mahlers Zeit, nach Geist und Leben wie Ich und Kaminski, seinen Ruhm verarbeitete Kehlmann in seinem Folgeprojekt -, und er lebt weiter in den zahlreichen Interviews, Werkstattgesprächen, im Aufsatz Wo ist Carlos Montúfar? und in der Poetikvorlesung Diese sehr ernsten Scherze. Selbst in sein Vorwort zu einer Neuedition von Charles Darwins Die Fahrt der Beagle baute er ein eigenes Humboldt-Zitat ein: „Die zweitgrößte Beleidigung des Menschen sei die Sklaverei, hatte Humboldt ausgerufen, die größte aber die Behauptung, er stamme vom Affen $a^{\circ "}(V M, \text { S. 238) })^{35}$. Dieses Zitat, welches sich auch im Film

35. - Daniel Kehlmann, « Die Finken und die Wilden. Einleitung », in: Charles Darwin, Die Fahrt der Beagle. Tagebuch mit Erforschungen der Naturgeschichte und Geologie der Länder, die auf der Fahrt von HMS Beagle unter dem Kommando von Kapitän Fitz Roy, RN, besucht wurden, Hamburg, marebuchverlag, 2006, S. 15, zitiert nach Frank Holl, ,„,Die zweitgrößte Beleidigung des Menschen sei die Sklaverei...' Daniel Kehlmanns neu erfundener Alexander von Humboldt", in: HiN. Internationale Zeitschrift für Humboldt-Studien, 2012, XIII, 25, S. 58. 
findet, wurde von Humboldt-Experten hinterfragt ${ }^{36}$. Im DVD-AudioKommentar nutzt Kehlmann wiederum die Gelegenheit, darauf Bezug zu nehmen und sich zu verteidigen: „Auch Humboldt-Forscher wissen nicht alles" (V 30:30).

Auch der Film erscheint in diesem Kontext als Experiment um ein lange betriebenes „Erfinden von Wahrheit" 37 sowie die von Kehlmann immer wieder gestellte Frage, bis zu welchem Grad man historische Persönlichkeiten fiktionalisieren dürfe ${ }^{38}$ und wie man über sie schreiben könne. In einem Interview fragte er: ,Wieso wirken historische Romane trivial, aber wieso wirkt nicht trivial, was etwa Eric Hobsbawm schreibt? Ein Fachhistoriker geht nicht zu nah ran an die Figuren"39. In Wo ist Carlos Montúfar? nennt er Stanley Kubricks Barry Lyndon als Musterbeispiel eines Historienfilms. Dieser Film erlangt seine Authentizität gerade nicht durch eine Abbildung des realen Lebens des 18. Jahrhunderts, sondern durch die Vorstellungen, die man sich heute davon mache ${ }^{40}$.

Aus beiden Thesen ergibt sich eine Erklärung für die überdeterminierte, überkonstruierte Bilderwelt des Films: Buck und Kehlmann erhalten einerseits die Mittelbarkeit und Distanz zum Geschehen aufrecht, welche für den Roman prägend ist, andererseits handelt es sich hier um eine Art Meta-Film über das Erzählen selbst. Dabei geht der Film einen Schritt weiter als das Buch: Tragen die Protagonisten im Roman die Reflexion über das Erzählen in den Text hinein und sind sich gar ihrer Fiktionalisierung bewusst, als sie zum Ende des Romans feststellen: „Unser Erfinder hat genug von uns“ (VM, S. 292 $)^{41}$, so stellt der Film die Frage nach der „Wahrheit“ weniger explizit, entscheidet sie aber umso eindeutiger: Hier siegen die Bilder jederzeit über die Figuren, die Inszenierung und der Erzähler Kehlmann dominieren die Geschichte überdeutlich. In einer Szene dreht sich das Bild, die Inszenierung schaltet zwischen Humboldt und Gauß hin- und her, als würde ein Fernsehzuschauer den Kanal wechseln (V 01:04:50).

36. - Vgl. ebd.

37. - Daniel Kehlmann, „Wo ist Carlos Montúfar?“, a.a.O., S. 14.

38. - Vgl. stellvertretend Daniel Kehlmann und Michael Lentz, "'Die Fremdheit ist Ungeheuer'. Gespräch über historische Stoffe in der Gegenwartsliteratur", in: Neue Rundschau, 2007; 118 (1): 33-47.

39. - Felicitas von Lovenberg, ,Ich wollte schreiben wie ein verrückt gewordener Historiker. Daniel Kehlmann im Gespräch“, in: Gunther Nickel (Hrsg.), Daniel Kehlmanns „Die Vermessung der Welt“. Materialien, Dokumente, Interpretationen, a.a.O., S. 32 .

40. - Daniel Kehlmann, „Wo ist Carlos Montúfar?“, a.a.O., S. 14.

41. - Markus Gasser, Das Königreich im Meer. Daniel Kehlmanns Geheimnis, a.a.O., S. 104. 


\section{Experiment und Abenteuer}

Die in dem Eingangszitat von Nabokov für die Übertragung seines Originaldrehbuchs verwendete Analogie zwischen Adaptation und Übersetzung ist ein in der Intermedialitätsforschung regelmäßig verwendetes Bild. Wie bei Übersetzungsleistungen hängt die „Wirkungsäquivalenz" von Literaturverfilmungen vom Verhältnis zwischen „Einbürgerung“ des Ausgangstexts und „Fremdhalten“ gegenüber der Vorlage $\mathrm{ab}^{42}$. Die Verfilmung der Vermessung der Welt nimmt hier eine mittlere Position zwischen beiden Polen ein: Sie hält recht genau fest an Handlungsabfolgen einzelner Szenen oder Dialoge, und befreit sich von der literarischen Vorlage, indem sie eigene thematische Schwerpunkte setzt und das Weltbild des Romans umdeutet.

Wie der Roman muss der Film als ein Experiment aufgefasst werden, welches seinen Platz im Gesamtwerk Kehlmanns findet. Während die Verfilmungen von Il nome della rosa oder Das Parfum den experimentellen Charakter der Vorlage eher überdecken, tritt dieser im Fall von Die Vermessung der Welt erst in den Vordergrund und widerspricht in Teilen der These, eine populäre Adaptation bedeute eine Simplifizierung des Ausgangstexts. Durch seine Schwerpunktsetzung erscheint der Film im Kontext des Werks Kehlmanns wie eine Weiterentwicklung eines im Roman diskutierten Darstellungsproblems.

Interessanterweise spiegeln sich die beiden in der Geschichte dargestellten Modelle der Weltvermessung, das deduktive und das induktive, auch in der Umsetzung beider Werke. Man kann bei Gauß am Küchentisch an den Autor Kehlmann denken, der den Roman in weiten Teilen in einem Haus mit Meerblick auf Sylt schrieb, angeblich während der Arbeit öfters in sich hineinlachend ${ }^{43}$, bei Humboldt an einen Arrangeur, der mit Bildern aus Ecuador und Görlitz, mit Toneffekten, Farbpaletten und Digitaleffekten jongliert und daraus einen Erzählkosmos zusammenfügt. So wie das Schreiben des Romans selbst eine Recherche- und Denkleistung darstellte, so impliziert der Film eine Suchbewegung und einen Moment des Aufbruchs und des Abenteuers: „Wenn wir einen Film über Forscher und Entdecker drehen, und es wird parallel gerade eine neue kinematographische Sprache erfunden, ist es naheliegend, dass wir diese auch benutzen und mit entwickeln wollen. Genau wie Humboldt und Gauß betreten wir Neuland mit 3D"44, sagt

42. - Vgl. Anke Bohnenkamp, „Literaturverfilmungen als intermediale Herausforderungen“, in: dies. (Hrsg.), Literaturverfilmungen, Stuttgart, Reclam, 2005, S. 36.

43. - Markus Gasser, Das Königreich im Meer. Daniel Kehlmanns Geheimnis, a.a.O., S. 84 .

44. - Wenka v. Mikulicz u. Michael Töteberg (Hrsg.), Die Vermessung der Welt. Das Buch zum Film, a.a.O., S. 183. 
Bucks Koproduzent Claus Boje im Buch zum Film. Ein dort abgedrucktes Foto, welches Louis Daguerres ersten Fotoapparat neben einer modernen 3D-Kamera zeigt, fasst zwei Aufbruchsmomente des Kinos zusammen: Die Geschichte des Bildes und seiner Darstellung ist noch lange nicht zu Ende. 
